\title{
CONTRIBUIÇÕES DO PROFISSIONAL DA EDUCAÇÃO FÍSICA NO CONTROLE DA HANSENÍASE A NÍVEL LOCAL
}

Gláucia Cristina de Castro

Aguinaldo Gonçalves

\section{Resumo}

Destacam-se inicialmente as bases formais que, na última década, vêm definindo com clareza a necessidade da recomposição da equipe de atuação no Sistema Único de Saúde. Nesse sentido, recuperam-se núcleos de experiências pioneiras de integração entre academia e serviços. Especificamente em relação ao controle da hanseníase a nível local, reconstrói-se o conhecimento das origens e características de prática de Educação e Saúde que exige, de seus executores, formação simultaneamente lúdica, pedagógica e atlética diferenciada, o que agrega evidências adicionais para a legitimidade da inclusão do profissional de Educação Física no cotidiano do setor saúde.

\section{Palavras-Chave}

Educação Física; Hanseníase; Contribuições multiprofissionais; Nível local.

\section{PHYSICAL EDUCATION PROFESSIONAL'S CONTRIBUTIONS IN THE LOCAL LEVEL CONTROL OF HANSENIASIS}

Gláucia Cristina de Castro

Aguinaldo Gonçalves

\begin{abstract}
Initially, it is highlighted the formal basis that has, through the last decades, clearly defined the need to recompose the acting team at Sistema Único de Saúde (Health Unique Service). In that regard, pioneering experience centers from the integration between academy and services are recovered. Specifically regarding the local level control, it is rebuilt origin and characteristics of a Health Education practice that requires from its performers a simultaneously differentiated ludic, pedagogic and athletic qualification, which adds additional evidences for the legitimation of the Physical Education professional's inclusion in the day-to-day of the health sector.
\end{abstract}

\section{Key-Words}

Physical Education; Hanseniasis; Multi-professional contributions; Local level.

\section{CONTRIBUCIONES DEL PROFESIONAL DE EDUCACIÓN FISICA PARA EL CONTROL DE LA ENFERMEDAD DE HANSEN A NIVEL LOCAL}




\section{Resumen}

Se destacan inicialmente las bases formales que en la última década vienen definiendo claramente la necesidad de recomponer el equipo de actuación en el Sistema Único de Salud. En este sentido se recuperan núcleos de experiencias pioneras a partir de la integración entre academia y servicios. Específicamente en relación al control de la enfermedad de Hansen a nivel local, se reconstruye el conocimiento de los orígenes y características de prácticas de Educación y Salud que exige de sus ejecutores, formación simultáneamente lúdica, pedagógica y atlética diferenciada, lo que agrega evidencias adicionales para la legitimidad de la inclusión del profesional de Educación Fisica en la cotidianeidad del sector de la salud.

\section{Palabras-Clave}

Educación Fisica; Hanseníase; Contribuciones multiprofesionales; Nivel local. 


\section{INTRODUZINDO CONTRIBUIÇÕES MULTIPROFISSIONAIS}

Refletindo a expansão internacional a respeito, o Conselho Nacional de Saúde, através da resolução no 218/97 em 06 de março de 1997, voltada a intensificação do trabalho interdisciplinar junto ao Sistema Único de Saúde, resolve reconhecer como profissionais de saúde de nível superior treze novas categorias, englobando o graduado em Educação Física - EF. Deu-se continuidade, desse modo, a inclusão da EF como parte integrante da equipe de saúde, através de experiências realizadas visando possibilitar melhor conhecimento e intervenção da relação Atividade Física - Saúde Coletiva, no controle de diversos agravos, tanto infecciosos como não transmissíveis, a hanseníase aí incluída.

Complementarmente, recente publicação do Ministério da saúde, a portaria 154, de 24 de janeiro de 2008, em seu artigo segundo, institui os Núcleos de Apoio a Saúde da Família, estabelece que os mesmos devem contar com equipe multiprofissional e classifica-os em duas modalidades, a primeira composta por no mínimo cinco profissionais, tais como acupunturista, professor de EF, nutricionista, ginecologista, psicólogo, entre outros e a segunda composta por, no mínimo, três profissionais, como farmacêutico, fisioterapeuta, professor de EF, assistente social, psicólogo e terapeuta ocupacional (BRASIL, 2008).

Especificamente sobre a atuação do profissional da EF, pontuam-se, no referido documento, as Ações de Atividade Física/Práticas Corporais a serem implementadas pelas equipes, para propiciar

[...] a melhoria da qualidade de vida da população, a redução dos agravos e dos danos decorrentes das doenças não transmissíveis, que favoreçam a redução do consumo de medicamentos, a formação de redes de suporte social e que possibilitem a participação ativa dos usuários na elaboração de diferentes projetos terapêuticos.

Sequentemente, conceitua-se que:

[...] as Práticas Corporais são expressões individuais e coletivas do movimento corporal advindo do conhecimento e da experiência em torno do jogo, da dança, do esporte, da luta, da ginástica. São possibilidades de organização, escolhas nos modos de relacionar-se com o corpo e de movimentar-se, que sejam compreendidas como benéficas à saúde de sujeitos e coletividades, incluindo as práticas de caminhadas e orientação para a realização de exercícios. Em face do caráter estratégico relacionado à qualidade de vida e à prevenção do adoecimento, as ações de Atividade Física/Práticas Corporais devem buscar a inclusão de toda a comunidade adstrita, não restringindo acesso apenas às populações já adoecidas ou mais vulneráveis.

A atualidade destas bases legais leva, neste texto, ao esforço de recuperação das recentes experiências encetadas em nosso meio a respeito, com o objetivo de explicitar, com clareza, a introdução de novas contribuições do profissional em questão ao controle da hanseníase a nível local, operando a partir da integração entre academia e serviços.

Conexões: revista da Faculdade de Educação Física da UNICAMP, Campinas, v. 8, n. 2, p. - , maio/ago. 2010.

ISSN: 1983-9030. 


\section{RECENTES EXPERIÊNCIAS}

Nesse sentido, cabe registro, desde pronto, dos trabalhos da Escola de Formação em Saúde da Família "Visconde de Sabóia" no município de Sobral, Ceará, órgão vinculado a Secretaria Municipal de Saúde e que desde 1997 atua como centro de educação permanente para os trabalhadores da saúde. Dentre as questões assim tratadas, está a valorização da equipe multiprofissional atuante no Programa Saúde da Família. Desde 2000, incluiu o profissional da EF, buscando estratégias junto a comunidade para a "prevenção de doenças, promoção da saúde e melhora na qualidade de vida da população”, como apontam Lucena et al. (2004). Na oportunidade relatam que a chegada da nova categoria ao grupo foi determinante na sensibilização de gestores locais para a necessidade de ações multidisciplinares nas Unidades Básicas de Saúde. Com fundamentação no perfil epidemiológico municipal e nos fazeres da EF, definiu-se proposta de ação centrada nesses profissionais.

Num primeiro estudo com a "Saúde e Arte: ensinando e encenando", os pesquisadores associaram-se a atores de rua e juntos compuseram peça teatral abordando temas como higiene, doenças sexualmente transmissíveis e gravidez precoce, a qual foi encenada para escolares, crianças e adolescentes, de escolas públicas (PRIAMO et al., 2005). Com a investigação "Atividade Física e Postural: Uma experiência interdisciplinar com grupos de idosos" visou-se atender número significativo de pessoas que procuravam o centro de saúde queixando-se de dores de origem osteomusculares. A partir disso, criou-se acompanhamento para realização de atividades como alongamento, relaxamento e educação postural (PAIVA; MANGUEIRA, 2006).

No interior do Grupo de Saúde Coletiva/Epidemiologia e Atividade Física da Unicamp (GSCEAFhttp://www.unicamp.br/fef/indexnew.htm), identifica-se linha de pesquisa específica voltada para Serviços de Saúde, desde 2002, embora nas demais temáticas podem-se encontrar ensaios que de alguma maneira também formalizam essa relação. Versam, peculiarmente, a respeito de índices antropométricos e risco à saúde de indivíduos obesos internados para tratamento em spa médico (CARVALHO; PADOVANI; GONÇALVES, 2003); programa de intervenção de hidroginástica para usuárias sedentárias, visando verificar efeito na qualidade de vida dessas pessoas (VICENTIN; GONÇALVES; PADOVANI, 2003), atividade física em contaminados com mercúrio (DEL VECCHIO; GONÇALVES, 2005); Economia Solidária, Esporte e Saúde relacionados na transformação de bairro em comunidade saudável, tendo a agência de saúde como palco de encontro e desencadeamento de ações de âmbito educacional, cultural e da saúde. De modo mais singular, podemos mencionar projeto que objetivou inserção de conteúdos da EF no cotidiano dos agentes comunitários

Conexões: revista da Faculdade de Educação Física da UNICAMP, Campinas, v. 8, n. 2, p. - , maio/ago. 2010.

ISSN: 1983-9030. 
de saúde, com a implementação em duas fases sucessivas, uma das quais compreendendo a adoção da metodologia de Grupo Focal: como resultado do seguimento efetivado, pode-se, em plano mais terminativo, sugerir que são eles capazes de compreender as contribuições da EF, por meio do estilo de vida, nas práticas sociais e que a maneira de viver de cada pessoa pode não ser opção individual (GONÇALVES;VICENTIN, 2004).

\section{O PROFISSIONAL DE EDUCAÇÃO FÍSICA NO PROJETO SÃO MARCOS: AS EXPLORAÇÕES EM HANSENÍASE}

Diretivamente em relação à hanseníase, o GSCEAF em seus mais de vinte anos de existência mantém-na como um de seus campos de estudo. Nesse período, produziu trinta e cinco trabalhos nesta temática, entre publicações em periódicos e anais de congressos, além das várias teses de mestrado e doutorado. Tais publicações estão alocadas em diferentes linhas de pesquisas desenvolvidas no interior do mesmo, conforme registrado na Tabela 1.

Tabela 1 - Distribuição de Frequiência de Publicações do GSCEAF Sobre Hanseníase

\begin{tabular}{lcc}
\hline \multirow{2}{*}{ Linha de Pesquisa } & \multicolumn{2}{c}{ Freqüência } \\
\cline { 2 - 3 } & Absoluta & Relativa \\
\hline Grupos Populacionais - Hansenianos & 20 & 57,1 \\
Saúde Coletiva e Atividade Física & 03 & 8,6 \\
Estudos Colaborativos Multicêntricos & 12 & 34,3 \\
Total & 35 & 100 \\
\hline
\end{tabular}

Basicamente, os ensaios pertencentes ao grupo Hansenianos referem-se à prevenção de incapacidades físicas (GONÇALVES et al., 1990; OLIVEIRA et al., 1990); lesões decorrentes de ocupação profissional relacionadas a atividade física (MONTEIRO et al., 1994); impacto da transição epidemiológica sobre a doença (GONÇALVES; PARIZOTTO; GONÇALVES, 1997); relação atividade física e prevenção de incapacidades (GONÇALVES; GONÇALVES, 2000) e educação em saúde (MANTELLINI; GONÇALVES, 2005).

Os estudos situados e conduzidos na perspectiva da segunda linha de pesquisa (Saúde Coletiva e Atividade Física) tratam fundamentalmente do diagnóstico precoce (GONÇALVES, 1989; GONÇALVES; GONÇALVES, 1990) e delineamentos de formas de tratamento (GONÇALVES; GONÇALVES; PADOVANI, 1998). Os designados por Estudos Colaborativos Multicêntricos, por envolverem pesquisadores 
e profissionais de diversas instituições, lidam com possibilidades e dificuldades na implantação de programas de controle (GONÇALVES et al., 1988; BONILHA et al., 1994); prevenção e tratamento de incapacidades (GONÇALVES et al., 1989; PEDROSO et al., 1989), centro de referência como núcleo de reabilitação (VIRMOND; DUERKSEN; GONÇALVES, 1989) e o emprego de dermatóglifos para detecção de grupo de risco (GONÇALVES et al., 1988).

Diante desse extenso espectro, importa focalizar os que contemplam a atividade física como meio para a prevenção de incapacidades. Neste caso, situa-se ensaio descritivo concebido visando gerar conhecimentos epidemiológicos e possibilidades de práticas da EF como recurso para tal fim. Os resultados observados, através de registros de dados de exame físico e anamnese da população acompanhada, apontaram, enquanto caminho possível, a pós- reabilitação e reinserção social, através de atividades adaptadas, tais como treino sensorial, dança, movimentos rítmicos, alongamento muscular e percepção cinestésica, fatores afetados diretamente pela hanseníase (GONÇALVES; GONÇALVES; PADOVANI, 2002). Em outro, encontramos a concernência entre lesões motoras e atividade física ocupacional pela investigação a respeito de lesões incapacitantes em pessoas com diferentes ocupações profissionais. Para tanto se buscou verificar presença, grau e localização de tais agravos, em doentes ativos e não ativos fisicamente. Após análise dos dados, revelou-se que os sedentários desenvolvem quadro inflamatório em maior proporção quando comparados aos ativos (MONTEIRO; GONÇALVES; PADOVANI, 2002). Com efeito, trata-se de dois estudos paradigmáticos complementares: enquanto um releva o sedentarismo na gênese da doença, o outro prospecta alternativas de superação da mesma através do movimento orientado e assistido.

\section{O TEATRO DE MÃO E HASTE (TMH)}

O destaque central aqui especificamente conferido é a projeto constituído por teatro interativo de bonecos, desenvolvido no interior do Centro de Saúde Santa Mônica, Campinas, SP, como recurso para controle da hanseníase a nível local, pelo estimulo ao diagnóstico precoce da doença: sensibilizando crianças e adultos para a questão da deficiência e hanseníase, proporciona intercâmbio de informações e leva ao movimento de construção/reconstrução de novos sentidos e significados.

Trata-se de trabalho implantado a partir do projeto The Kids on the Block, existente nos Estados Unidos. O grupo americano construiu histórias para tratar de grande diversidade de temas/assuntos, tais como: separação dos pais, HIV, obesidade, epilepsia, deficiências (mental, auditiva, física), racismo, além de outros (KOTB,

Conexões: revista da Faculdade de Educação Física da UNICAMP, Campinas, v. 8, n. 2, p. - , maio/ago. 2010. 
2006). Para cada situação existem personagens e roteiros diferenciados, sempre participando integrante que não apresenta nenhum tipo de agravo físico, social ou emocional. Utiliza adaptação da técnica japonesa chamada bunraku, representação teatral clássica, interpretada por bonecos. $\mathrm{O}$ termo deriva do artista Uemura Bunrakuken que construiu em 1805 pequeno teatro em Osaka. O espetáculo tradicional é constituído por narrador, tocador de "shamisen" (instrumento de cordas semelhante ao banjo) e os manipuladores. Nenhum cenário ou cortina esconde os manipuladores; apenas roupa e capuz negros são empregados. Ao contrário de exibições de fantoches ou de marionetes, não cria a ilusão de que agem ou falam por si mesmos. Para tanto é necessário que o apresentador tenha total conhecimento da prática para que se possam realizar diferentes movimentos, suportar o peso do boneco e manter a postura do mesmo, estando ele em posição desconfortável (GIROUX; SUSUKI,1991).

Os bonecos utilizados são chamados "de mão e haste". Têm cerca de 1 metro de altura e utilizam acessórios cênicos, originais ou miniaturas de objetos, tornando-se, assim, reais e distintos. Em cada exibição participam de dois a quatro personagens que são manipulados por duas pessoas. Em termos de Brasil, em 1984 foi estudada a possibilidade de adaptação do programa, vindo para cá, dois anos após profissional para realizar o primeiro treinamento de que surgiu Ana Melo, menina de 11 anos, portadora de hanseníase. O roteiro e o desenho foram criados no Brasil por Thomas Ferran Frist, idealizador da Sociedade para Reabilitação e Reintegração do Incapacitado (Sorri), entidade que lhe albergou institucionalmente a iniciativa.

Esta é uma organização não governamental surgida em 1972, com o apoio da agência internacional American Leprosy Mission. Após dois anos de maturação, estruturou-se plano de ação para reabilitação de ex-doentes. A região de Bauru foi escolhida para a implantação, por considerar-se a existência do antigo Leprosário Aimorés, hoje Instituto Lauro de Souza Lima. Pretendia-se reorganizar os sistemas segregados e centralizadores que caracterizavam o tratamento existente neste período. Desde então, a organização tem modificado sua prática em função das mudanças ocorridas na área. Com isso, atualmente existem oito unidades no Brasil: São José dos Campos, Campinas, Bauru, Sorocaba e Litoral Norte no estado de São Paulo, Salvador-Bahia e Parauapebas-Pará, desenvolvendo atividades muito diversas, de acordo com a comunidade a que atendem (SORRI, 2006).

Já encenado também nos Estados Unidos e no Chile, o TMH compõe-se, enquanto dinâmica de palco, de duas partes: a primeira compreende o conhecimento do tema através da historia exibida e a segunda, interação com

Conexões: revista da Faculdade de Educação Física da UNICAMP, Campinas, v. 8, n. 2, p. - , maio/ago. 2010. 
a platéia, mediante diálogo entre personagens e expectadores. A "criança" sem hanseníase apresenta relacionamento positivo alicerçado na diversidade. É ela quem fala dos preconceitos, dúvidas e receios. No decorrer da história, tem-se a oportunidade de explicar o que é o agravo, suas causas, tratamento e cura. $\mathrm{O}$ momento da realização das perguntas é usado para estimular o debate, visando esclarecer dúvidas e impropriedades conceituais. Tais questões são responsáveis por dinamizar a interação e posterior ressignificação de conceitos pela interlocução crianças - personagens, bem como explicitar atitudes tanto negativas como positivas frente à situação proposta (CASTRO, 2005).

\section{A ESPECIFICIDADE DO PROFISSIONAL DA EDUCAÇÃO FÍSICA}

Em recente publicação (CASTRO; GONÇALVES, 2009), teve-se a oportunidade de elaborar o reconhecimento da singularidade do perfil do profissional de Educação Física, por basear-se em tripé presente apenas em sua formação, constituído pelos componentes lúdico, pedagógico e atlético. Essa a razão técnica fundamental da identificação, entre nós, do TMH com tal educador. Com efeito, chama a atenção a peculiaridade das demandas envolvidas nessa pratica, passíveis de serem operadas, sobretudo a partir da formação do professor de Educação Física, quanto às respectivas habilidades motoras. No plano aplicado geral, já foram elas objeto de detalhado estudo em nosso grupo (GONÇALVES, 2004), de sorte que se torna oportuno focá-las, ainda que perfunctoriamente, em suas relações específicas com o TMH.

De fato, a força, valência física necessária a toda e qualquer atividade do ser humano, em termos de TMH demanda pedagogia e aprendizagem diferenciadas, considerando-se que se trata de suportar o peso dos bonecos, mantê-los em pé e com postura adequada. A flexibilidade, influenciada por aspectos endógenos e exógenos, com ápice de desenvolvimento da infância para a adolescência, mostra-se relevante para a manipulação dos bonecos, principalmente quando da "fala" dos mesmos. A velocidade, referida aos movimentos das diversas ações cotidianas, caracteriza o deslocamento próprio dos personagens. A resistência, definida como tempo de atividade física praticada com certa intensidade, no TMH manifesta-se em todos os trinta/quarenta minutos de duração das apresentações, imprescindível na manutenção das posições dos apresentadores, sobretudo quando desconfortáveis. A coordenação motora, relação dinâmica, harmoniosa e uniforme das demais capacidades biomotoras na realização de uma tarefa, no TMH é determinante para que todos os outros aspectos trabalhem a favor da sincronização para execução das expressões gestuais constituintes do espetáculo. 


\section{CONSIDERAÇÕES FINAIS}

A partir de todas essas concepções e práticas de suporte e com o apoio estrutural e financeiro do Fundo de Apoio ao Ensino, à Pesquisa e à Extensão, através da solicitação 812/07 (FAEPEX, Edital de Projetos Anuais na Área de Extensão Comunitária, PREAC, UNICAMP), desenvolveu-se intervenção que atingiu 207 crianças e adolescentes, compreendidos na faixa etária entre 7 e 16 anos, de ambos os sexos, moradores do Complexo São Marcos, Campinas,SP e participantes de oficinas e atividades realizadas em período complementar ao da escola formal. No decorrer das seis apresentações, nove indivíduos pediram encaminhamento para avaliação dermatológica no Centro de Saúde. Dada a especificidade do processo, a análise desta fase está implicando em nova investigação ampliada em andamento no interior de nosso Grupo de pesquisa, tendo-se em conta que dois deles puderam ser avaliados, dos quais em um a doença foi diagnosticada. Ora, como na região, de aproximadamente vinte mil habitantes, existe registro ativo de dois casos em tratamento, pode-se constatar que houve acréscimo de 50\% no indicador epidemiométrico de prevalência (CASTRO; GONÇALVES, 2005).

Acumularam-se, assim, evidências a favor da participação do profissional de Educação Física no interior das equipes de Saúde incumbidas de prevenção e controle de afecções endêmicas em nosso meio, face à especificidade das habilidades desenvolvidas em sua formação específica.

\section{REFERENCIAS}

BONILHA, V. F. R. et al. Projeto experimental de controle da hanseníase na cidade de Bauru-SP: avaliação do primeiro ano de implementação. Hans Int, v. 19, n. 2, p. 11-18, 1994.

BRASIL. Ministério da Saúde. Portaria $n^{\circ}$ 154, de 24 de janeiro de 2008, que cria os Núcleos de Apoio à Saúde da Família - NASF. Brasília, 2008.

CARVAlHO, P. J. A.; PADOVANI, C. R.; GONÇALVES, A. Evolução na classificação de índices antropométricos, relacionados a fatores de risco à saúde, no tratamento da obesidade em spa médico. In: CONGRESSO INTERNACIONAL DE EDUCAÇÃO FÍSICA E MOTRICIDADE HUMANA, 3., 2003, Rio Claro. Anais... Rio Claro: UNESP, 2003.

CASTRO, G. C. Transformação social por meio do Movimento Sorri:do isolamento à inclusão. In: GONÇALVES, A.; GUTIERREZ, G. L.; VILARTA. R. (Org.). Gestão da qualidade de vida na empresa. Campinas, IPES Editorial, 2005. p.65-83. 
CASTRO, G. C.; GONÇALVES, A. Recortes da experiência do teatro interativo como estratégia de educação em saúde em hanseníase. In: CONGRESSO BRASILEIRO DE HANSENOLOGIA, 10., 2005, Recife. Anais... Recife, 2005.

CASTRO, G. C.; GONÇALVES, A. Intervenção e formação em Educação Física com destaque à Saúde. Motriz, v. 15, n. 2, p. 374-382, 2009.

DEL VECCHIO, F. B.; GONÇALVES, A. Avaliação subjetiva da qualidade de vida em pessoas contaminadas por mercúrio em ambiente urbano-industrial pela aplicação do SF36. In: ENCONTRO CIENTÍFICO DE EDUCAÇÃO FÍSICA E ESPORTE/METROCAMP, 1., 2005, Campinas. Anais... Campinas, Metrocamp, 2005.

GIROUX, S. M.; SUZUKI, T. Bunraku: um teatro de bonecos. São Paulo: Perspectiva, 1991.

GONÇALVES, A. Pesquisa epidemiológica em hanseníase: necessidades e perspectivas atuais. In: CONGRESSO BRASILEIRO DE HANSENOLOGIA, 7., 1989, Rio de Janeiro. Anais... Rio de Janeiro, 1989. (Org.). Conhecendo e discutindo saúde coletiva $e$ atividade física. Rio de Janeiro: Guanabara Koogan, 2004.

. PEDROSO, M. ; OLIVEIRA, S. ; BACARELLI, R. Dificuldades e sugestões para a implantação de atividades de prevenção de incapacidades em serviços de controle da hanseníase no Brasil. Rev Bras Med, 45,n.10, p. 422-426, out,1988.

GONÇALVES, A. et al. Geração de função discriminante linear em dematóglifos para detecção de grupos de risco em hanseníase. An Bras Dermatol, Rio de Janeiro, v. 63, n.5, p. 395-400, set./out., 1988.

GONÇALVES, A. et al. Prevenção e tratamento das incapacidades físicas em hanseníase: estudo da evolução em nosso meio, com ênfase na avaliação de determinantes de sua efetividade. Revista Brasileira de Medicina, v. 46, n. 7, p.269-284, jul,1989.

GONÇALVES, A. Prevenção e tratamento das incapacidades físicas em hanseníase II: estudo da evolução em nosso meio, com ênfase na avaliação de determinantes de sua efetividade. In: Congresso Brasileiro de Epidemiologia 1., 1990, Campinas. Anais... Campinas, 1990.

GONÇALVES, A. ;VICENTIN, A. P. M. Promovendo e exercendo a economia solidária através da saúde e do esporte: a experiência da Unicamp no Jardim São Marcos, Campinas, In: Encontro de Economia Solidária, 2., 2004, São Paulo. Anais... São Paulo, 2004.

GONÇALVES, G.; PARIZOTTO, N.A.; GONÇALVES, A. A hanseníase no contexto da transição epidemiológica III: perspectivas da aplicação de laserterapia de baixa intensidade em úlceras específicas. In: 
CONGRESSO DO COLÉGIO DE HANSENOLOGIA DOS PAISES ENDÊMICOS, 4., 1997, Foz de Iguaçu. Anais ... Foz do Iguaçu, 1997.

GONÇALVES, G.; GONÇALVES, A.; PADOVANI, C. R Laserterapia de baixa intensidade (L.B.I.) em úlceras hansênicas em serviços locais de saúde (SILOS): primeiros resultados da experiência brasileira, In: CONGRESSO BRASILEIRO DE FISIOTERAPIA ESTÉTICA, 1., 1998, Campinas. Anais... Campinas, 1998.

GONÇALVES, G.; GONÇALVES, A.; Incapacidades em hanseníase e atividade física: mapeando e apontando possibilidades em unidade sanitária local. In: CONGRESSO BRASILEIRO DE SAÚDE COLETIVA, 6., 2000, Salvador. Anais... Salvador, 2000.

GONÇALVES, G.; GONÇALVES, A.; PADOVANI, C. R. Promoção a saúde a partir da epidemiologia das incapacidades física em hanseníase: caminhando na cidadania em unidade de referência do Sistema Único de Saúde. In: CONGRESSO BRASILEIRO DE EPIDEMIOLOGIA,5., 2002, Curitiba. Anais... Curitiba, 2002.

GONÇALVES, N.; GONÇALVES, A. Avaliação de resultados de diferentes tecnologias alocadas ao diagnóstico precoce da hanseníase. Rev Saúde Dist Fed, v.1, n.1, p. 16-22, jan./mar., 1990.

KOTB. Kids on the block: an educational puppet troupe. Disponível em: www.kotb.com. Acesso em: 8 de setembro de 2006.

LUCENA, D.; BORGES, K. E. L.; AGUIAR, M. A.; ALCANTARA, F.; MADRUGA, J. G.; LOMEO, R. A inserção da Educação Física na estratégia saúde da família em Sobral/CE. SANARE: revista de políticas públicas , ano 5, n. 1, p. 87-91, jan./mar. 2004.

MANTELLINI, G. G.; GONÇALVES, A. Incapacidades físicas em hanseníase: coisa do passado ou problema do futuro? In: CONGRESSO BRASILEIRO DE HANSENOLOGIA, 10., 2005, Recife. Anais... Recife, 2005. MONTEIRO, H. L. et al. Saúde e urgência em educação física e esportes III: estudo de retoanálise sobre atividade física e incapacidades em hanseníase. In: REUNIÃO ANUAL DA SBPC, 46., 1994, Vitória. Anais... Vitória, 1994.

; GONÇALVES, A. PADOVANI, C. R. Atividade física ocupacional e lesões sensitivo-motoras nas hanseníase: investigação a partir de estudo transversal híbrido no Instituto Lauro de Souza Lima, Bauru SP. In: SIMPÓSIO INTERNACIONAL DE TREINAMENTO DESPORTIVO, 2002, João Pessoa. Anais... João Pessoa, 2002.

OLIVEIRA, S. et al. Adequação de procedimentos de Instrumentalização de dados em prevenção e tratamento de incapacidades físicas em hanseníase. In: CONGRESSO BRASILEIRO DE DERMATOLOGIA, 45., junho, 1990.

Conexões: revista da Faculdade de Educação Física da UNICAMP, Campinas, v. 8, n. 2, p. - , maio/ago. 2010.

ISSN: 1983-9030. 
PAIVA, G.; MANGUEIRA, J. Atividade física e postural: uma experiência interdisciplinar com grupos de idosos. In: EXPOSIÇÃO DE SAÚDE PÚBLICA DE FORTALEZA, 2., 2006, Fortaleza. Disponível em: http://www.esf.org.br/publicacoes/expoesp2006/atividade_fisica.php. Acesso: 03 dez. 2007.

PEDROSO, M. et al. Prevenção e tratamento das incapacidades físicas em hanseníase no Brasil - estudo de rsico profissional específico para a doença. Han Int, v. 14, n. 2, p. 112-119, dez.1989.

PRIAMO, V. et al. Saúde e arte: ensinando e encenando. IN: EXPOSIÇÃO DE SAÚDE PÚBLICA DE FORTALEZA,1., 2005, Fortaleza. Disponível em: http://www.esf.org.br/publicacoes/expoesp2005/ saude_arte.php. Acesso em: 03 dez. 2007.

SORRI. Sistema Sorri: uma história de trabalho na promoção dos direitos da pessoa com deficiência. Disponível em: www.sorri.com.br/sistemas.htm. Acesso: 16-17 ago. 2006.

VICENTIN, A. P. M.; GONÇALVES, A.; PADOVANI, C. R. Efeito da hidroginástica sobre a flexibilidade de mulheres sedentárias excluídas da população economicamente ativa: intervenção a partir de recomendações do Colégio Americano de Medicina Desportiva. In: SIMPÓSIO INTERNACIONAL DE CIÊNCIAS DO ESPORTE, 26., 2003, São Paulo. Anais... São Paulo, 2003.

VIRMOND, M.; DUERKSEN, F.; GONÇALVES, A. Relato e avaliação de experiência brasileira na reabilitação de hansenianos. Lepr Rev, v. 60, p. 214-220, 1989.

\section{GLÁUCIA CRISTINA DE CASTRO}

Profa. Educação Física, Mestra

\section{AGUINALDO GONÇALVES}

Médico Sanitarista, Professor Titular, Saúde Coletiva e Atividade Física, Faculdade de Educação Física (FEF/Unicamp)

\section{Referência do artigo}

\section{ABNT}

CASTRO, G. C.; GONÇALVES, A. Contribuições do profissional da educação física no controle da hanseníase a nível local. Conexões, v. 8, n. 2, p. 133-145, 2010.

\section{APA}

CASTRO, G. C., GONÇALVES, A. (2010). Contribuições do profissional da educação física no controle da hanseníase a nível local. Conexões, 8(2), 133-145. 


\section{VANCOUVER}

Castro GC, Gonçalves A. Contribuições do profissional da educação física no controle da hanseníase a nível local. Conexões, 2010; 8 (2): 133-145.

\section{Recebido em:}

Aceito para publicação em: 